\title{
A SYSTEM OF SUBROUTINES FOR ITERATIVELY \\ REWEIGHTED LEAST SQUARES COMPUTATIONS
}

\author{
David Coleman* \\ Paul Holland** \\ Neil Kadent \\ Virginia Klema* \\ Working Paper No. 189 \\ National Bureau of Economic Research, Inc. \\ 575 Technology Square \\ Cambridge, Massachusetts 02139
}

\section{July 1977}

This research was supported by the National Science Foundation under Grants DCR75-08802 and MCS76-11989.

*National Bureau of Economic Research, Inc., Computer Research Center, 575 Technology Square, Cambridge, Massachusetts 02139.

*HEducational Testing Service, Rosedale Road, Princeton, N. J. 08540.

+Department of Computer Science, University of Toronto, Toronto, Canada M5S I A7. 


\section{ABSTRACT}

A description of a system of subroutines to compute solutions to the iteratively reweighted least squares problem is presented. The weights are determined from the data and linear fit and are computed as functions of the scaled residuals. Iteratively reweighted least squares is a part of robust statistics where "robustness" means relative insensitivity to moderate departures from assumptions. The software for iteratively reweighted least squares is cast as semi-portable Fortran code whose performance is unaffected (in the sense that performance will not be degracled) by the computer on operating-system environment in which it is used. An $\ell_{1}$ start and an $\ell_{2}$ start are provided. Eight weight functions, a numerical rank determination, convergence criterion, and a stem-and-leaf display are included.

Key Words and Phrases: least squares, data analysis, mathematical software, portability, linear algebra, curve fitting, robust estimation, weight functions.

CR Categories 5.14 and 5.5 
TABLE OF CONTENTS

Introduction . . . . . . . . . . . . . . . . . . 1 Section 1... . . . . . . . . . . . . . . . 3 Section 2 . . . . . . . . . . . . . . . . . 6 Section 3 . . . . . . . . . . . . . . . . 8 Acknowledgments. . . . . . . . . . . . . . . 26 References . . . . . . . . . . . . . . . . 27 
Introduction

The purpose of this paper is to describe a system of Fortran subroutines written as modular mathematical software to solve the iteratively reweighted least squares problem. The software includes documentation for use and flow of control as comments in the subroutines. The specifications from which the software was written are contained in [12]. The collection of subroutines uses orthogonal factorizations by Householder transformations or the singular value decomposition from EISPACK II [ 7 ] to compute the $\ell_{2}$ start and iterations for reweighted least squares. CLI [ 2 ] computes the $l_{1}$ start, an overdetermined solution in the $\ell_{1}$ norm.

The computational tools that we provide include an interactive driver, eight weight functions, John Tukey's stem-and-leaf display [15, 9] the diagonal of the "hat" matrix [10] which is the projection matrix $\mathrm{P}_{A}$ effectively computed as $U \Sigma \Sigma^{+} \mathrm{U}^{\mathrm{T}}$ from the singular value decomposition [7] or $\mathrm{QQ}^{\mathrm{T}}$ from $\mathrm{QR}$, the Householder transformations. Optionally, the $\ell_{2}$ condition of the matrix, the weights, residuals, and the convergence criterion can be displayed. Two forms of equilibration are also provided [16].

The usual statistics information to display the number of observations, number of variables, maximum diagonal element of the "hat" matrix, condition number of the weighted data matrix, the maximum absolute value of the residuals, and the minimum weight is optionally available. There is an option to provide the (weighted) sum of squared residuals, and the sum of absolute residuals. Also available is the (weighted) R-squared statistic, the (weighted) standard error, and the (weighted) F statistic. 
The software is presented in the form of a basis tape suitable for use by the Fortran converter [1] from IMSL to produce target Fortran code for CDC, Burroughs; Honeywell, PDP-10, and Univac machines. The source code is long precision IBM code acceptable to the Fortran converter. The PFORT verifier [14] was used to check the software,

We do not discuss the theoretical properties of iteratively reweighted least squares or the tuning constants for the weight functions in this paper. Rather we refer the reader to Holland and Welsch [II] for such information,

The organization of this paper is as follows. Section 1 defines the iteratively reweighted least squares problem. Section 2 describes the selection of rank for the data matrix and the re-weighted data matrix. Section 3 gives some numerical results. The weight functions are listed in Table 1 of Section 1. The subroutines for the computation are listed in Table 2 of Section 3. A copy of the subroutine to compute one of the weight functions, i.e., the Biweight weight function, is listed at the end of Section 3. 


\section{Section I}

The method of least squares has been the primary technique for fitting models to data for many years and is versatile and numerically stable when computationally stable methods are used [13]. Despite its central role in the past, much work has been done by statisticians to improve least squares in the sense of getting more information about the data than is available from just the least squares solution or from the matrix factorizations that are used to obtain it.

The area of work that our software addresses is robust regression which is aimed at analyzing and improving the behavior of least squares estimation when the disturbances are not well-behaved. We focus our attention on one of the computational procedures for robust linear regression, iteratively reweighted least squares.

Consider the model $\mathrm{b}=\mathrm{Ax}+\mathrm{r}$ where $\mathrm{b}$ is an mxl vector of observations, $A$ is an mxn data or design matrix, $x$ is an nxl vector of parametens, and $r$ is an $m x l$ vector. The notation $b=A x+r$ corresponds to the statistical notation $y=X \beta+\varepsilon$ where $y$ is $n \times I, X$ is $n \times p, \beta$ is $p \times I$, and $\varepsilon$ is $n \times l$.

The ordinary least squares problem is $\min _{x} \sum_{i=1}^{m}\left(\left(r_{i}(x)\right) / s\right)^{2}$ where $r$ is a vector of residuals $b$ - Ax, and $s$ is a constant or fixed scale. The weighted least squares problem is $\min _{\frac{1}{x}} w_{i=1}\left(\left(r_{i}(x)\right) / s\right)^{2}$ which is solved by using ordinary least squares with $w^{1 / 2} A$ and $w^{1 / 2} b$ where $W$ is a diagonal matrix of weights that are functions of scaled residuals. The iteratively reweighted least squares problem assumes a start 
$\hat{\mathrm{x}}^{(0)}$, which can be obtained from $\ell_{2}$, ordinary least squares, least absolute residuals, that is to say, the overdetermined solution in the $l_{1}$ norm, previous iterations of iteratively reweighted least squares, or a start specified by the user, Given $\hat{x}$, the problem is iterated to obtain the least squares solution, $\hat{x}^{(k+1)}=\left(w^{(k+1)^{1 / 2}}\right)^{A}+\left(w^{(k+1)}\right)^{1 / 2} b$ where the diagonal matrix $\mathrm{W}$ is computed as a function of scaled residuals. The residual scaling function we use is the maximum absolute deviation, $i, e .$, the median of the absolute values of the non-zero residuals. The modularity of the software makes readily possible the inclusion of additional residual scaling functions such as the inner-quartile-range of the residuals. The software to compute the weights includes the eight weight functions listed in Table 1.

To test convergence of iteratively reweighted least squares we use the convergence criterion suggested by John Dennis [4], After the $k^{\text {th }}$ iteration, we compute a scale-independent measure of the gradient, $A^{T} r$, where $r$ is the residuals $b-A x$, which is

$$
\left(\left(\left(w^{(k+1)}\right)^{1 / 2} A_{j}\right)^{T}\left(\left(w^{(k+1)}\right)^{1 / 2} r^{(k)}\right)\right) /\left\|\left(w^{(k+1)}\right)^{1 / 2} A_{j}\right\|_{2}\left\|\left(w^{(k+1)}\right)^{1 / 2} r^{(k)}\right\|_{2}
$$

where $\|\cdot\|$ is the Euclidean norm,

The problem of iteratively reweighted least squares is a problem in optimization in the sense that one is minimizing a function of scaled residuals, 
Table 1

Weight functions (where $u=$ scaled residual), range and default tuning constants.

Name $\quad \underline{w(u)}$

ANDREWS

CAUCHY

FAIR

HUBER

LOGISTIC

TALWAR

WELSCH

$$
w_{A}(u)=\left\{\begin{array}{l}
\sin (u / A) /(u / A) \\
0
\end{array}\right.
$$

Range

$$
w_{B}(u)=\left\{\begin{array}{ll}
{\left[1-(u / B)^{2}\right]^{2}} & |u| \leq B \\
0 & |u|>B
\end{array} \quad B=4.685\right.
$$

$A=1.339$

$|u|>\pi A$
Tuning Constant

$C=2.385$

$F=1.400$

$|u| \leq H$

$\mathrm{H}=1.345$

$|\mathrm{u}|>\mathrm{H}$

$\mathrm{L}=1.205$

$w_{L}(u)=(\tanh (u / L) y(u / L)$

$w_{T}(u)=\left\{\begin{array}{l}1 \\ 0\end{array}\right.$

$|u| \leq T$

$|u|>T$

$T=2.795$

$R=2.985$ 
Section 2

Starting points for the iterations include $\ell_{2}$, ordinary least squares and $l_{1}$, the overdetermined solution in the $\ell_{1}$ norm [2] which corresponds to least-absolute-residuals regression, The $\ell_{2}$ start and iterations subsequent to the $l_{1}, l_{2}$, or user-supplied start are computed by orthogonal fractorizations, i.e, Householder transformations or a combination of Householder transformations and the singular value decomposition.

The way in which we decide to use the QR (Householder transformations) or a combination of $\mathrm{QR}$ and MINFIT [ 7 ] (least-squares solution by singular value decomposition) needs some explanation. Frequently the data matrix, A, in the statistical model $b=A x+r$ has some variables (colums) that are close in the numerical sense to being linear combinations of other colums of A. Since such a situatior may occur the numerical rank [ 8 ] of A must be determined before proceeding with the least-squares computation. The numerical rank should be determined by the user, and the determination of rank should be made with respect to the certainty of the data. Since the rank must be determined at every iteration (reweighting may down-weight rows, i.e., observations, to the extent that the effective deletion of observations creates rank degeneracy) it is necessary to estimate the condition of the weighted $A$ as inexpensively as possible. For the $\ell_{2}$ start and for all iterations after any start we default to $a \cdot Q R$ factorization with colum pivoting. Unless $A$ is exactly singular the completion of the $Q R$ factorization of $A$ provides the upper triangular factor $R$ whose condition is that of $A$. The condition estimate using $R \quad[3]$ is only $O\left(n^{2}\right)$ operations, gives a reliable measure of the ill-conditioning of $\mathrm{A}$, and is used to determine whether $\mathrm{QR}$ is computationally sufficient or whether the computationally more expensive singular value decomposition, MINFIT, is necessary. 
We strongly believe that the user should determine the rank of his data or design matrix by inspecting the singular values of $A$ (which are the same as those of $R$ ). However we provide a conservative default determination of rank associated with the condition of the matrix at each iteration relative to the square-root of the precision of the computing machine that is used. Explicitly, the condition estimate of $R$ as obtained by [3 ] is an estimate of the largest and smallest singular values, $\sigma_{\max }$ and $\sigma_{\min }$, of $A$. If the ratio $\left(\sigma_{\min } / \sigma_{\max }\right)<\varepsilon^{1 / 2}$ where $\varepsilon$ is the relative precision of the arithmetic of the computing machine, the computation is continued by using the singular value decomposition. When the singular value decomposition is used the number, $k$, of singular values such that

$$
\sigma_{1} \geq \sigma_{2} \geq \ldots \sigma_{k}>0, \sigma_{k+1}=\ldots \sigma_{n}=0 \text {, is }
$$

determined from the certainty of the data or from the square root of the precision of the computing machine, whichever is larger. 
Section 3

The software to compute the solutions to the iteratively reweighted least squares problem consists of 17,500 lines of code, comments, and documentation for use. The 17,500 lines includes all of the software that is required for the interactive driver and its options for use plus a selection of test matrices. The software includes "help" conmands to give on-line information to users. The interactive dniver is designed to operate effectively on any computer system that permits the transmission of four characters to and from a teminal. The subroutines, however, can also be used in a batch environment. With the exception of CLl, all of the software is designed to be processed by the Fortran converter. We have included a selection of test matrices including those from [5]. We chose this particular collection of matrices because of the widespread use of [5] as a reference and text. The matrices are cast in integer form and then assigned as floating point numbers to insure that there is uniform input to a variety of computing machines.

The name and a brief description of the subroutines that are needed for all options of the iteratively reweighted least squares problem are listed in Table 2 of this section.

Selected results from one of the weight functions, Biweight, and the terminal session used to compute the results applied to [ 6 ] follows Table 2. 
The data matrix given in $[6]$ is

$$
A=\left[\begin{array}{rrr}
1 & .499 & 11.1 \\
1 & .558 & 8.9 \\
1 & .604 & 8.8 \\
1 & .441 & 8.9 \\
1 & .550 & 8.8 \\
1 & .528 & 9.9 \\
1 & .418 & 10.7 \\
1 & .480 & 10.5 \\
1 & .406 & 10.5 \\
1 & .467 & 10.7
\end{array}\right] \quad B=\left[\begin{array}{l}
11.14 \\
12.74 \\
13.13 \\
11.51 \\
12.38 \\
12.60 \\
11.13 \\
11.70 \\
11.02 \\
11.41
\end{array}\right]
$$

and has singular values $31.6, .109$, and .395 . 
Table 2

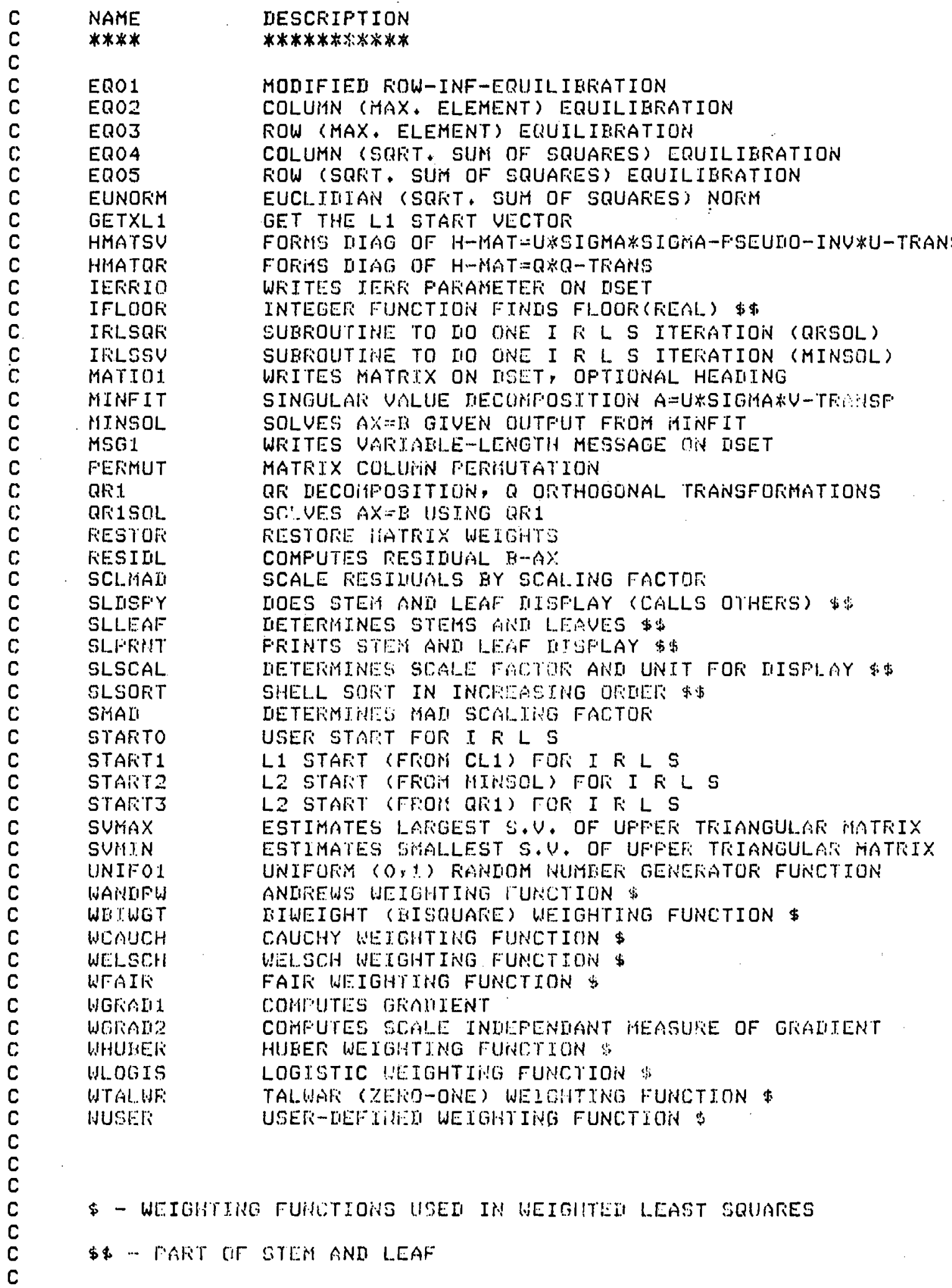


Table 2 con't.

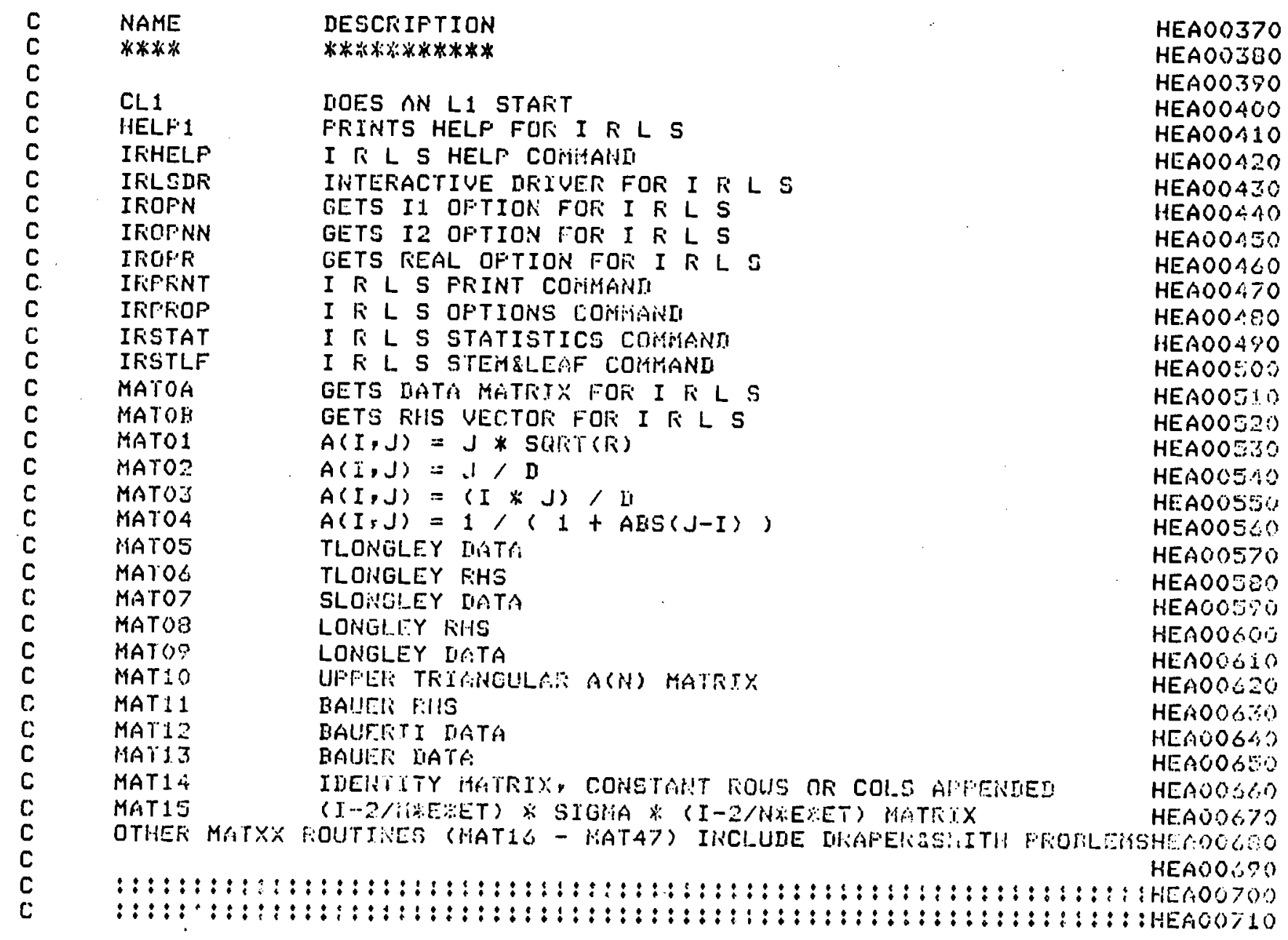


Sample Terminal Session with some Numerical Results

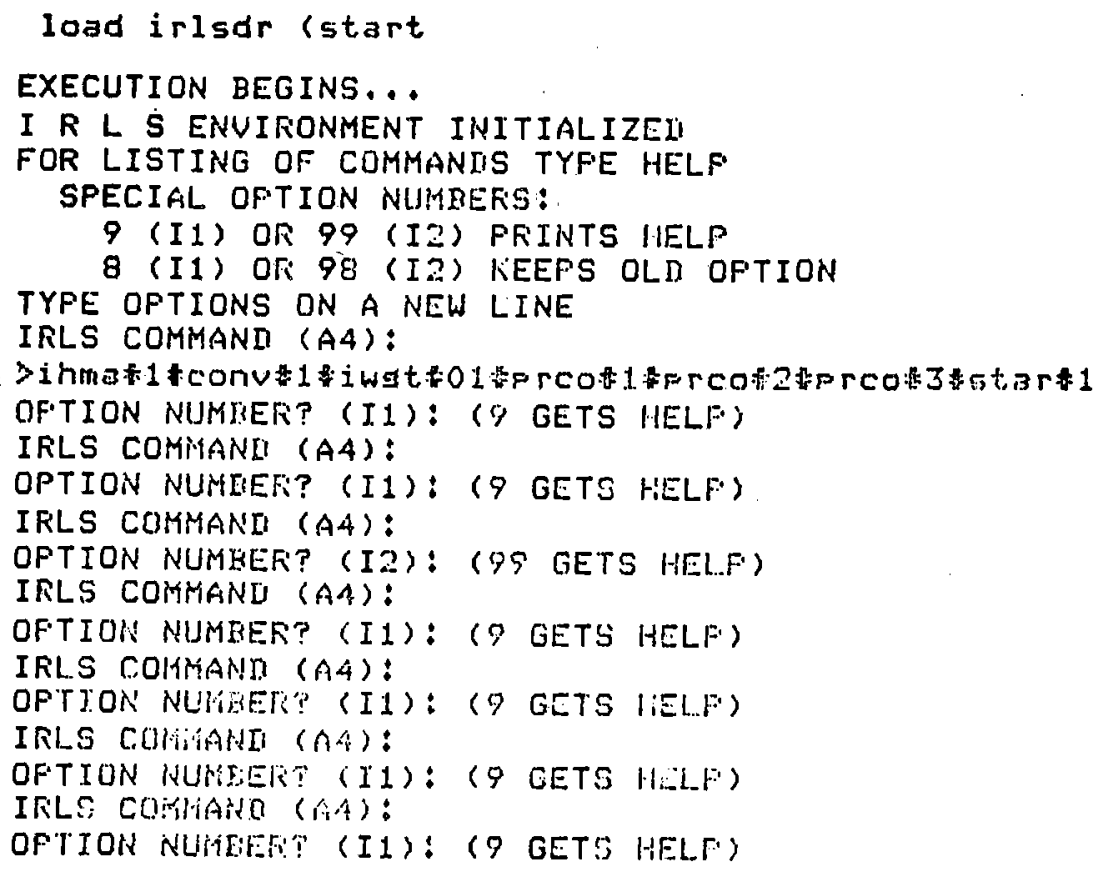

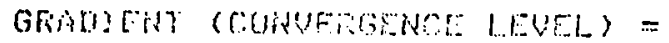

$0.25059693400 \quad 0.294495711+000.39530042100$

IHLS Combrob $(\hat{\mathrm{n} A})$ :

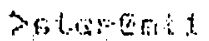

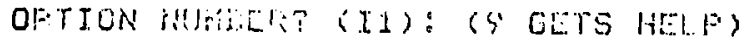

$=:=z=z:=: A:=$

Fil:STr!? S!..S

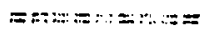


1

$$
\text { ( UNIT }=0.10000-01)
$$

$\begin{array}{llll}2 & -2 & \text { I } & 2 \\ 2 & -1 . & \text { I } \\ 2 & -1 & \text { I } & \\ 4 & -0 . & \text { I } & 96 \\ 4 & -0 & I & \\ 3 & 0 & \text { I } & 134 \\ 3 & 0 . & \text { I } & 77\end{array}$

1
LO I $\quad-0.5978$

$$
\text { HI I } 0.3859
$$

TEFR $=0$ FOR FESIDUALS

IRLS COMMAHR (AA):

istem: 2

OFTION NUIEEFT' (II): ( 9 GETS HELF)

$==+\cdot=====$

W-MATRIX

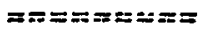

STEH-AIVIMLFEF DISFIAAY, $N=10$

1

LO I $\quad 0.55 .47$

( UNIIT $=0.10000 \cdots 01)$

2

FIS

5 I

a. I

$9 \mathrm{I}$

$T$ T 2

F I

517

9. I $89 \%$

101000

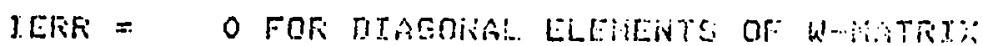

IRLS CONHW:

$>\operatorname{sectin} 3$

OFTIUA RIRBETE (XI): ( 9 GLTS HEIF)

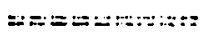

II-HATRIX

$==5:=:=5::=$

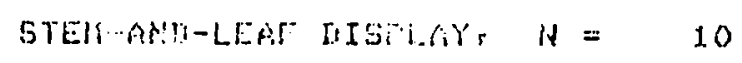

( VNIT $=0.1000 \% 01$;

11 I $A$ 


$\begin{array}{llll}2 & 1 \cdot & \text { I } & 8 \\ 5 & 2 & \text { I } & 134 \\ 5 & 2 . & \text { I } & 58 \\ 3 & 3 & \text { I } & 2 \\ 2 & 3 . & \text { I } \\ 2 & 4 & \text { I } & 4\end{array}$

1

HI I $0.680 \mathrm{E}$

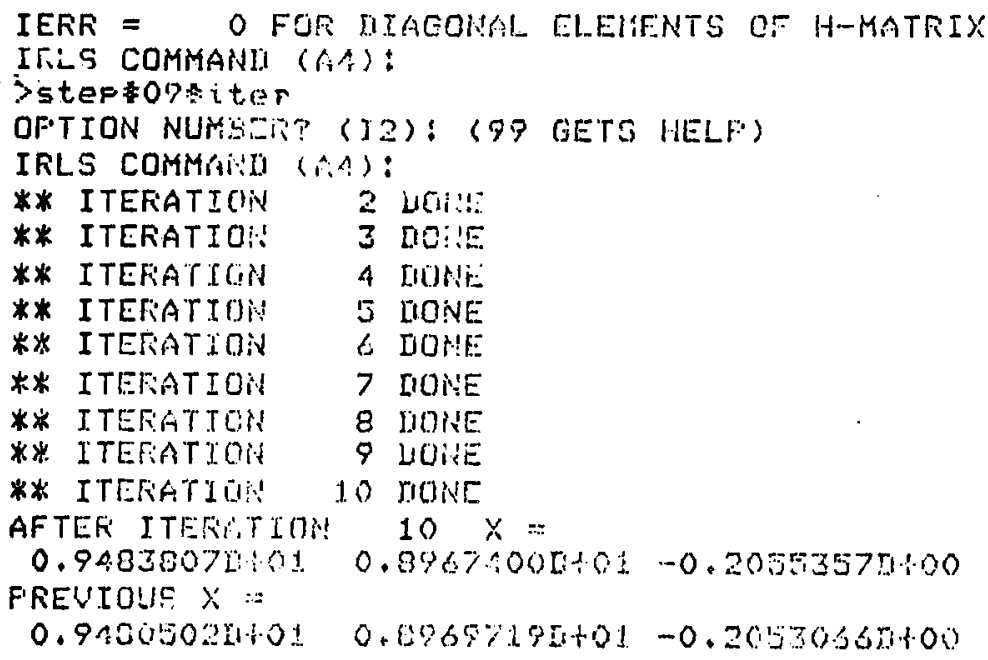
I FiESTMUAi_(I)
$1-0.5370,50 n+00$
0.81651757101
$0.38557825-01$
$-0.99 \div 62.4001001$
$5-0.227162616+00$
60.41620 sintoo
$70.970521011 . .01$
$0.69966 .1512 \cdots 02$
WITAG(I)
0.077966501400
HIIIAG $(T)$
$0.99257340+00$
$0.2601124 \mathrm{~L}+00$
0.90034301100
$0.2529899[1+00$
$0.9890925 \mathrm{~m} 100$
0.44614625160
0.94248430400
$0.65772025+60$
0.80707360100
$0.2377027[1+00$
0.90502565100
$0.11898510+00$
90.5353276501
0.99457660400
0.27201000100
$0.99600311+00$
$0.1957716 \pi+60$
0.21571 .785400
$10-0.02350502-01$
$0.95063 a_{10}+100$
$0.23554 \div 16600$

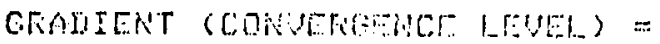

$-0.30166801-03-0.70330920-63-0.102620051-02$ 
IRLS COMMAND (AI4):

Smaxi $\neq 20 *$ step $10 * i$ ter

OPTION NUMBER? (I2): (99 GETS HELF)

IRLS COMMANII (A4):

OPTION NUMEER? (I2): ( 99 GETS HELF)

IFLS COMMANN (AA):

** ITERATION 11 IONE

** ITERIATION 12 DONE

* ITERATION 13 DONE

** ITERATION 14 DONE

** ITERATION 15 IIONE

** ITERATION 16 IIONE

* * ITERATION 17 DONE

** ITERATION 18 DONE

* ITERATION 19 LIONE

* ITERATION 20 IIONE

AFTEK ITEFATION $20 \quad X=$

$0.9488481 n+01 \quad 0.8964120[1+01-0.2058557[1+00$

FREUIOUS $\mathrm{X}=$

$0.94834650+01 \quad 0.8964131[1+01-0.2050586[1+00$

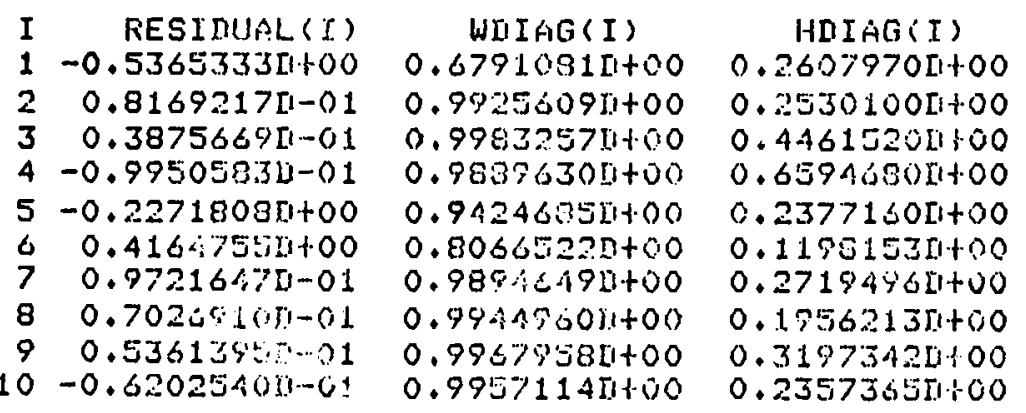

GRADIENT (CONUERGEMCE LEVI-I.) =

$-0.42635791-05-0.38069675-60-0.49603561-05$

IRLS COIBIAND (AIA):

>stentis

OFTIOH HUThER? (I1): ( 9 GETS HELP)

$==\pi====\approx=$

FESIIUUALS

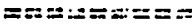

STEM-RIU-LEAF IIISPI_AY, $N=10$

1

LO I $\quad-0.5365$

(UIIIT $=0.1000[1-01\rangle$

2
2
2
4
4
5
5

$-2 \quad 1 \quad 2$

-1 . I

$-1 \quad I$

-0 . I 96

$-0 \quad I$

0 I 3

0. I 5799 


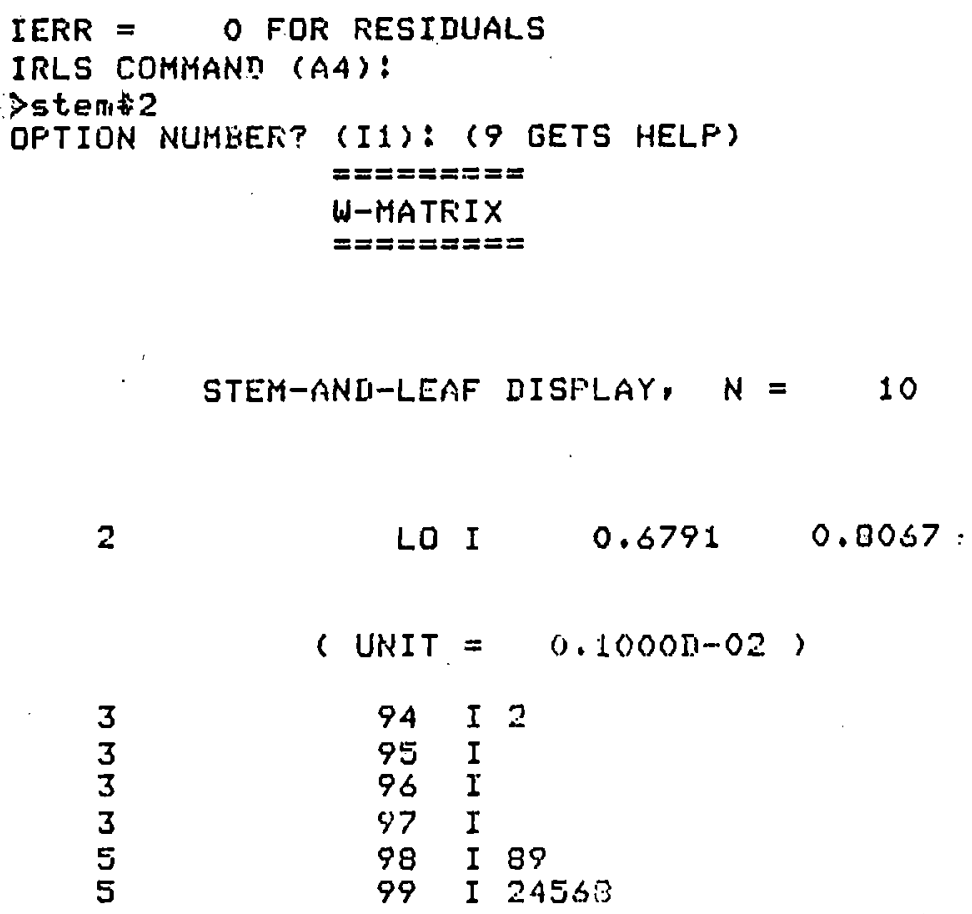

IEIT: $=$ O FOR ITACONAL ELEMEYTS OF W-MATEIX

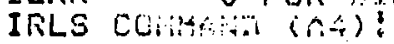

istention

OPTION NUREET? (II): ( 9 GETS HELP)

$== \pm= \pm= \pm=2-$

H-MATRIXX

$=:==\pi:=\therefore=$

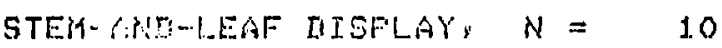

$$
(\text { Wirt }=0.1000 \mathrm{H} \cdots 01)
$$

$\begin{array}{llll}1 & 1 & \text { I } & 1 \\ 2 & 1 & \text { I } \\ 4 & 2 & \text { I. } & 33 \\ 3 & 3 & \text { I } & 57 \\ 3 & 3 & 1 & 1\end{array}$

2

HI I $\quad 0.4062 \quad 0.6595$

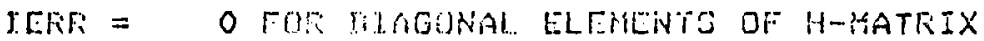

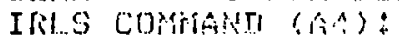

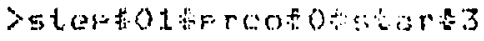

OFT TUW HUWEF' (T2): (79 GETS HELF)

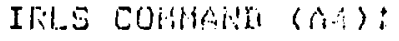

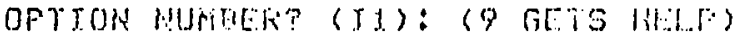

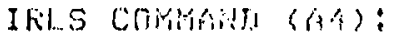

OPTICH NUABLE: ( $J 1$ ) : (9 GLTS MELP) 


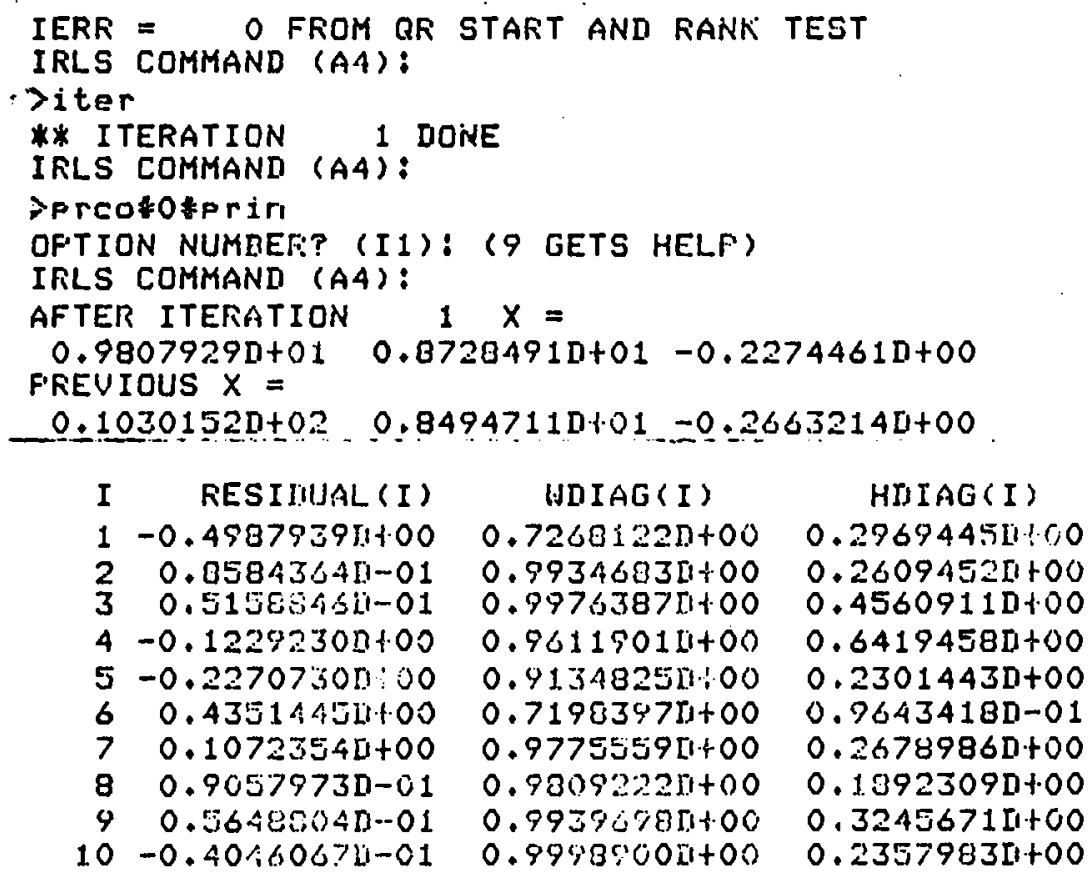

GRAIIENT (CONVETGEINCE LEVEL) =

$$
0.386123251-010.25549135-010.62805230-01
$$

IRLS COMMANR (H):

Deterist

OFYION NUMEETS (TI): ( 9 GETS HELF')

$=== \pm====$ :

RESINLALS

$=\approx=\approx==\approx=:$

STEM-ABM-LEAF LISFLAY, $W=10$

1

LOI $\quad-0,1980$

$($ UIVIT $=0.100011-01)$

$\begin{array}{llll}2 & -2 & I & 2 \\ 2 & -1 & I & \\ 3 & -1 & I & 2 \\ 3 & -0 & I & \\ 4 & -0 & I & 4 \\ 4 & 0 & I & \\ 4 & 0 . & I & 55 E 0 \\ 2 & 1 & I & 0\end{array}$

$1 \quad H I I \quad 0.4351$

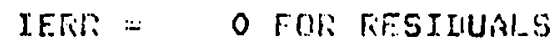

Ifils COHERATI (AA):

$>$ st, tan?

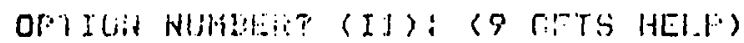
$=: \because=:=:=: \cdots:=:=$ W-intiatx : 
STEM-AND-LEAF DISFLAY, $N=10$

$$
2
$$

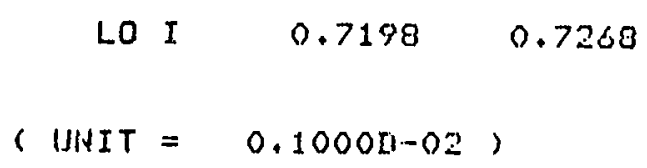




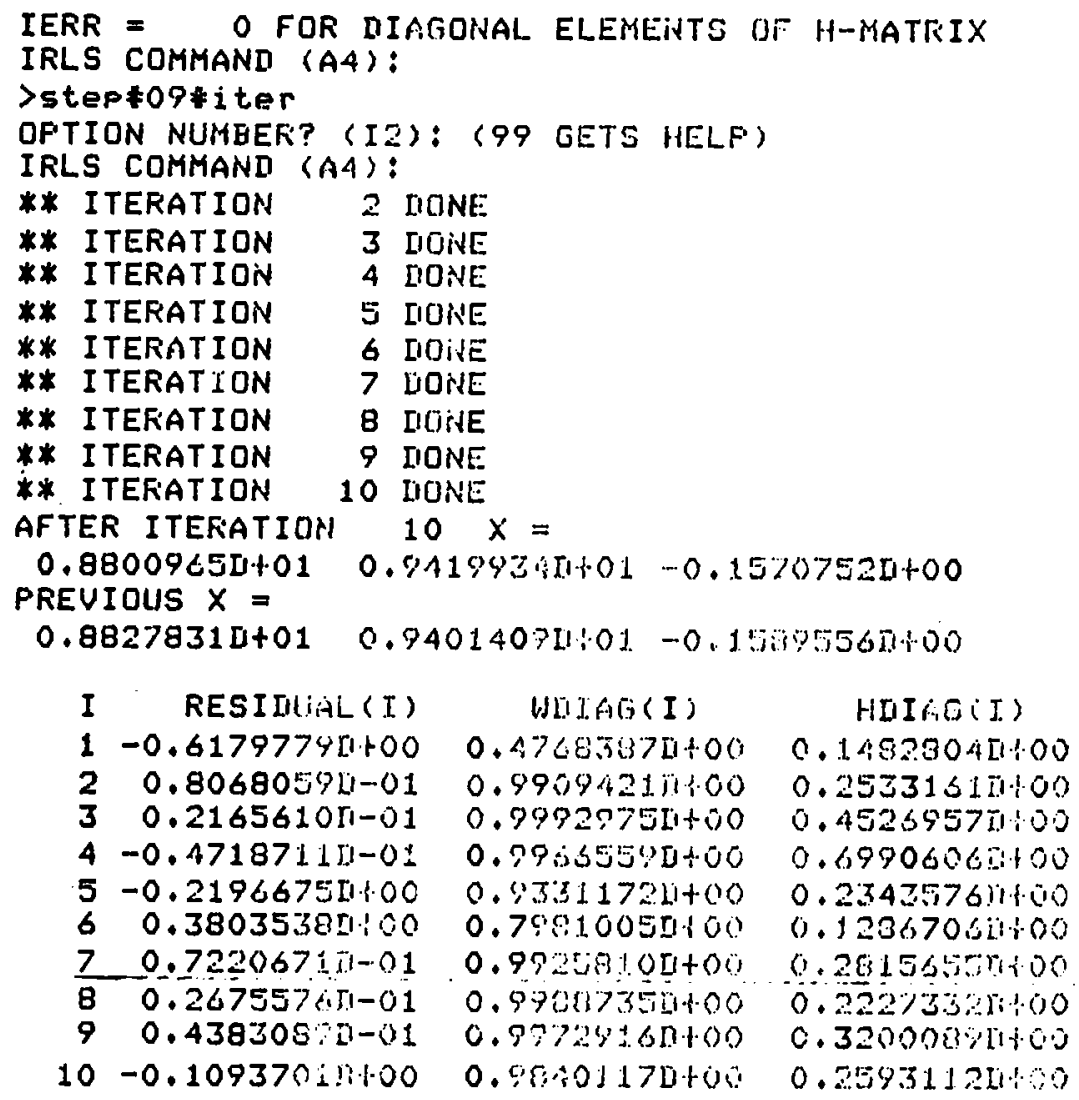

GRADIENT (CORUERGEINCE I.EVEL) =

$0.77033245-0 ?, 0.676990 .39-02 \quad 0.91670301-02$ 


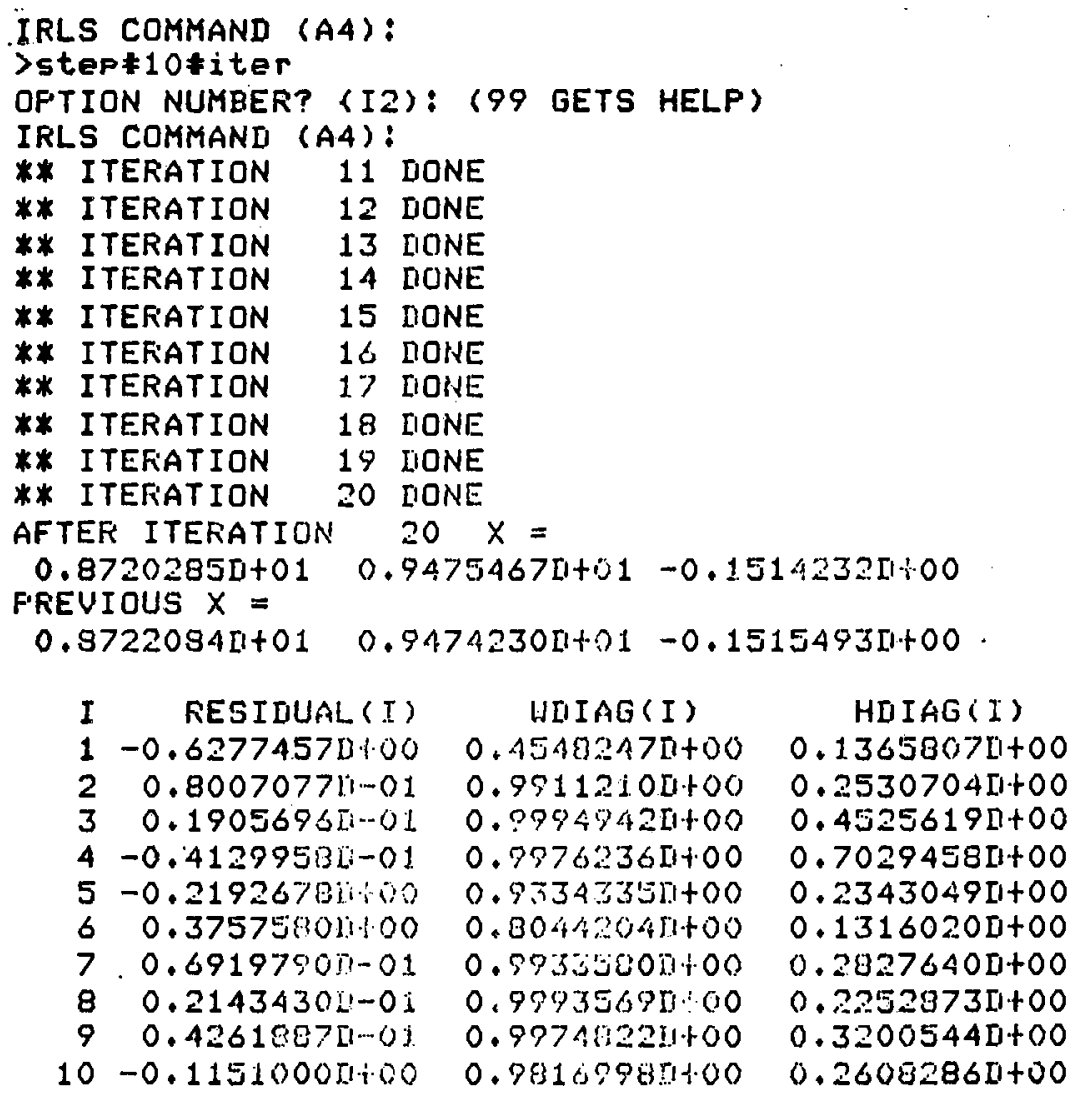

GRADIENT (CONUERCENCE LEUEI) =

$$
\begin{array}{lll}
0.519974251-03 & 0.45613870-03 & 0.620275211-03
\end{array}
$$

$$
\begin{gathered}
\text { LO I } \quad-0.6277 \\
\text { (UNTT }=0.1000 \text { LI-01) }
\end{gathered}
$$

$\begin{array}{llll}2 & -2 & I & 1 \\ 2 & -1 & I & \\ 3 & -1 & I & 1 \\ 3 & -0 & I & \\ 4 & -0 & I & 1 \\ 3 & 0 & I & 124 \\ 3 & 0 . & I & 68\end{array}$

$1 \quad H I$ I 0.3758 


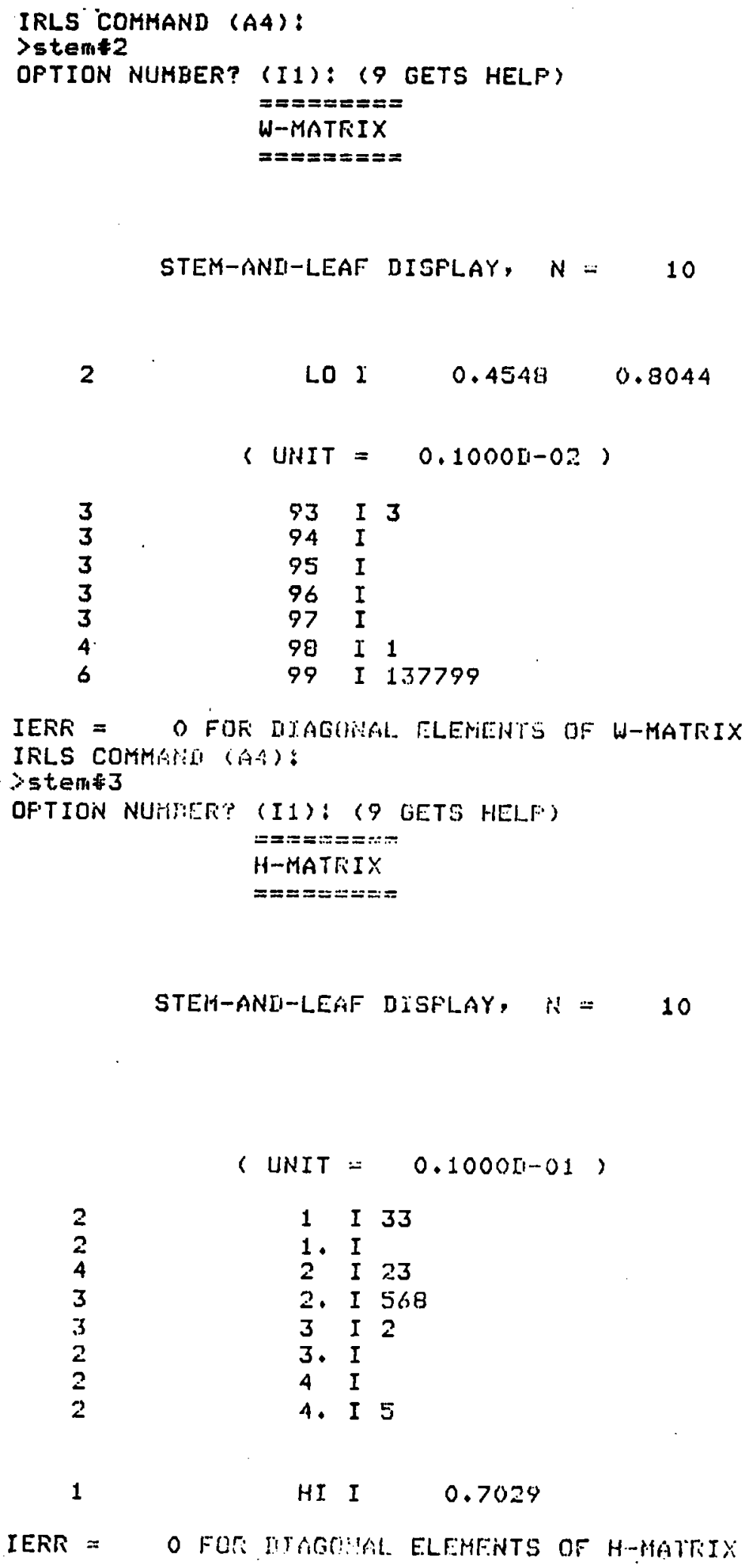


In conclusion we show the listing of one subroutine - the Biweight weight function. The software on tape for the iteratively reweighted least squares problem is available from the Algorithns Distribution Service. The authors of this paper are responsible for any modifications that subsequent use may show to be necessary. 
c

*****F'ARATETERS:

INTEGER $N$

REAL *8 $U(N)$, CONST, SQW(N)

c

*****LOCAL VARIAELES:

INTEGER I

REAL*8 OFLIM,UFETA,U1,FROI

C *****FUNCTIONS:

C

REAL $* 8$ IIABS
WBI00010

WEIO0020

WB 100030

WEI 100040

WEIO0050

WEI00060

WEIUOOO 0

WBI 100080

WEI TOOOSO

WEIO0 100

WEIOO 110

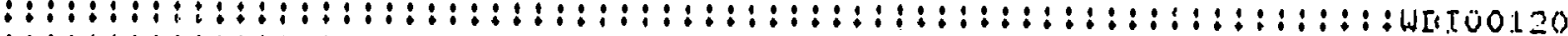

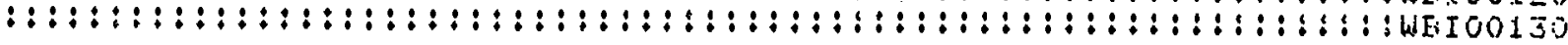

*****FURPOSE:

THIS SURFICUTINE PRONUCES THE SOUARE ROOTS OF THE WEIGHTS

DETERIIISIE TSY THE INFUT VECTOF U OF FREUIOUSLY COMFUTEII

SCALEI RESIIUALS AHII THE TIUEIGHT (EISQUARE) WEIGHT FUNCTIDN.(1)

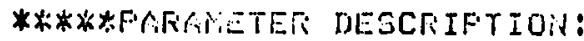

ON IHEIT:

$N$ BUST BE SET TO THE NUREER OF ELEMENTS IN THE WROTORS U MAS SQW:

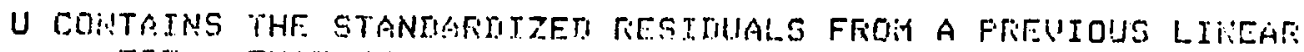
FIT. THAT JS, U(J) = Fi(I) / S WHERE RiSI) IS THIS I-TH RESITUAL FFOM A LINESR FIT, Fi(I) = Y(J) - YFITTEM(I),

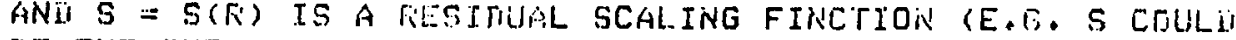

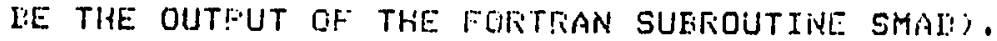

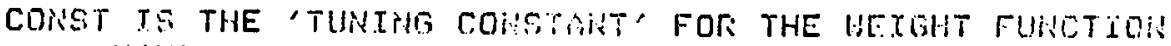
W(U). COOHST UUST EE PUGTTIVE (SEE OFFLICATTUN ANJ USAGE RLESTELCTIOLIS).

ON OUTFLUT:

SQI CCNTTAINS A VECTOH OF THE SQUARL ROETS OF THF UT.IEHTS

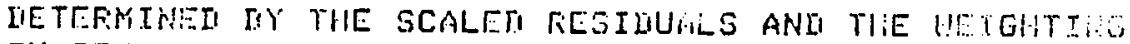
FUiNCTIEU,

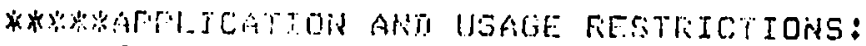

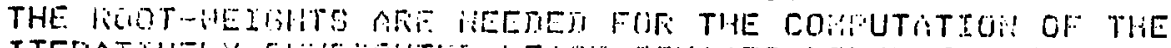

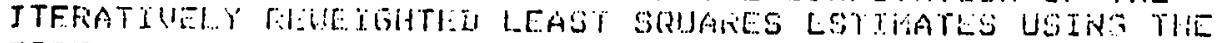

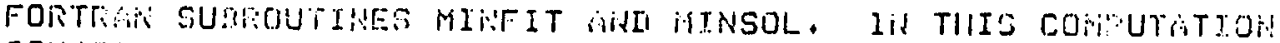

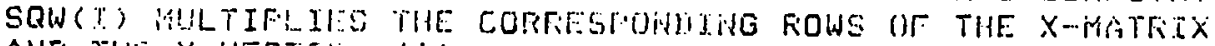
ANI THE Y-UECTCH. (1)

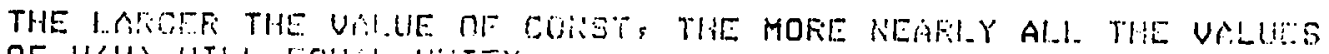
OF U(U) UILL EQUGL. LINTTY.

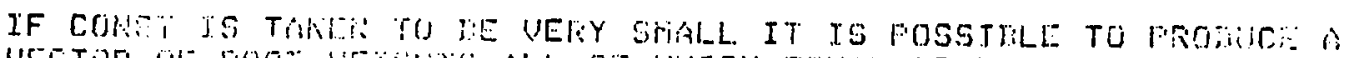

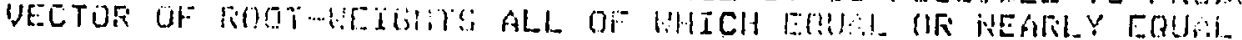

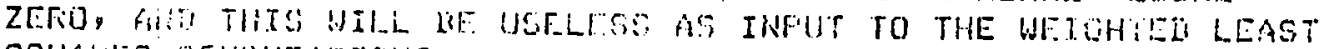

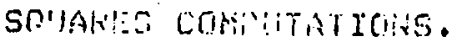

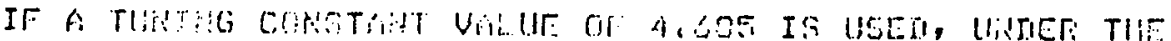

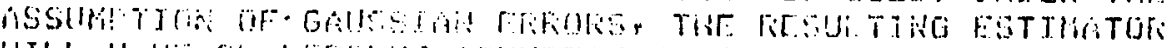

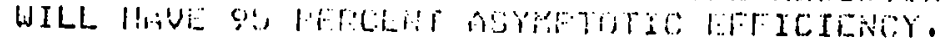

WEI UOU 4.0

WEXOOIIO

WEI UO 160

WEIO01\%0

WETOOL8O

Wถั IÜ01\%0

NETOŨ230

WEIOO 210

WEIOOZ2\%

WE $1002: 0$

WHIO0:?

HEJ00250

WEIT00260

WEIU0 270

HBIOO230

WEI OO290

HEJU0 000

UEIO031.0

WEI00

WBI I003:0

WETUOS, 00

WHEIUO 360

HEI ON 370

WBID0300

WEIU0590

U15:0 60

WET IUO $: 30$

WE 100435

UET00तio

WEIOOA5C

WEIDO 40

WEI00\%70

WBIOUASO

WEI IOS 90

WEICOSic

WBI00 10

WISI DOS?:

WEI I005S

UTi I 1005 :

HBIUOSLO

WEIOOE6?

WEIOOFO

HDT OUSBO

HEI 00550

WEr roos:

WETOOGI:

HBTOت̈6?:
WII U0 030

WETOOADO 


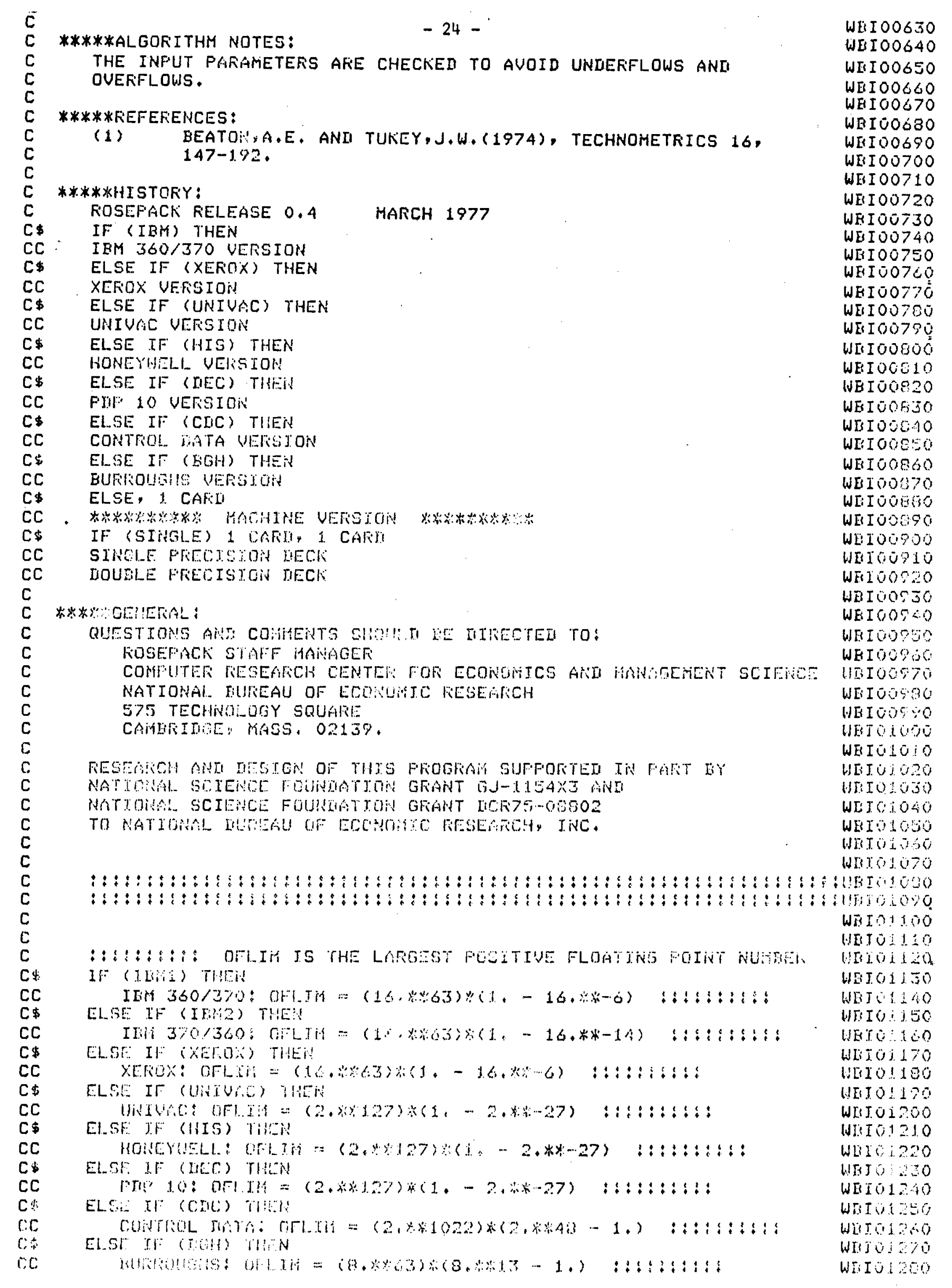




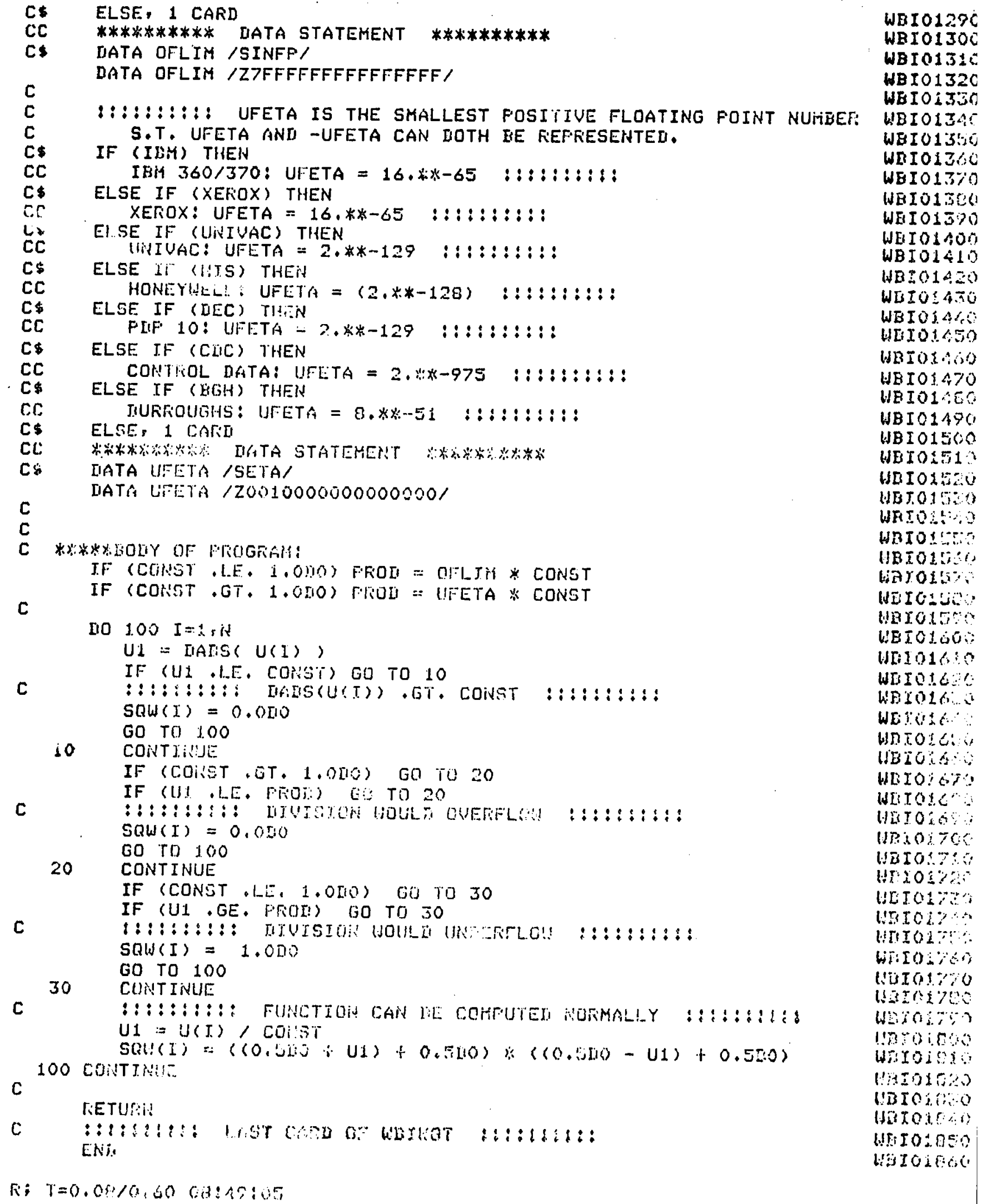




\section{Acknowledgments}

The authors are grateful to many people for their assistance in doing this work. Especially we thank John Dennis, Gene Golub, and Roy Welsch for their helpful suggestions. David Hoaglin and Stan Wasserman provided the software for the stem-and-leaf display which gives a useful summary of computational results, Richard Bartels made available the software for the $l_{I}$ start. Alan Cline, Cleve Moler, G. W. Stewart, and J. H. Wilkinson shared with us their technique for estinating the condition number of a matrix. David Gay, with valuable advice from David Hoaglin. on random number generators, wrote the code for the condition estimate.

Michael Sutherland and Richard Becker helped to check the software on CDC and Honeywell computers. Douglas Raynor ran several versions of the code on the PDP 10. T. J. Aind and Ed Battiste supplied the Fortran converter from TMSL.

Paul Velleman, after using the interactive driver from its own documentation, made valuable suggestions concerning documentation for use. Maurice Herlihy and Steve Peters independently used the software, checked. parts of the program and documentation for use. Sandra Moriarty has provided technical assistance throughout the period in which this work was done. 


\section{$\underline{\text { References }}$}

1. Aird, T. J., "The Fortran Converter User's Guide," IMSL, 1975.

2. Bartels, R., and Conn, A., "Linearly Constrained Discrete $\ell_{1}$ Problems," Johns Hopkins University, Technical Report \#248, June 1976.

3. Cline, A., Moler, C., Stewart, G. W., and Wilkinson, J. H., "On an Estimate for the Condition Number of a Matrix," informal manuscript, 1977.

4. Dennis, J., private communication, June 1976.

5. Draper, N, R., and Smith, H., Applied Regression Analysis, John Wiley and Sons, Inc., 1966.

6. Draper, N. R., and Stoneman, D., "Residuals and Their Variance Patterns," Technometrics, 8, 1966, p. 695-699.

7. Garbow, B. S., Boyle, J. M., Dongarra, J. J., and Moler, C. B., Matrix Eigensystem Routines - EISPACK Guide Extension, Springer-Verlag, Lecture Notes in Computer Science, 51, 1977.

8. Golub, G., Klema, V., and Stewart, G. W., "Rank. Degeneracy and Least Squares Problems," University of Maryland, TR-456, 1976, Stanford University, STAN-C5-76-559, 1976, National Bureau of Economic Research, Inc., Working Paper 165, 1977.

9. Hoaglin, D. C., and Wasserman, S., "Automating Stem-and-Leaf Displays," National Bureau of Economic Research, Inc., Working Paper 109, 1975.

10. Hoaglin, D. C., and Welsch, R. E., "The Hat Matrix in Regression and ANOVA," Harvard University, Department of Statistics, Memo. NS 341 , December 1976. 
II. Holland, P., and Welsch, R., "Robust Regression Using Iteratively Reweighted Least Squares," Communications in Statistics: Theory and Methods, 1977.

12. Kaden, N., and Klema, V., "Guidelines for Writing Seni-portable Fortran," National Bureau of Economic Research, Inc., Working Paper 130, 1976.

13. Lawson, C. L., and Hanson, R. J., Solving Least Squares Problems, Prentice-Hall, Inc., 1974.

14. Ryder; B. G., The Fortran Verifier: User's Guide, Computing Science Tech. Report 12, Bell Telephone Labs., 1975.

15. Tukey, J. W., Exploratory Data Analysis, Addison-Wesley, 1977.

16. Van Der Sluis, A., "Condition, Equilibration and Pivoting in Iinear Algebraic Systens," Number. Math. 15 (1970). 
Errata Sheet to Working Paper \#189.

Table of Contents: replace lines 2, 3, and 4 with

Section 1, Iteratively Reweighted Least Squares . . . 3

Section 2, Selecting the Rank of the Data Matrix. . . 6

Section 3, Some Numerical Results . . . . . . . . 8

page 1, Introduction: paragraph 2, line 5: replace

"Optionally" with "Displaying".

paragraph 2, line 6, 7: replace "can be displayed" with "is an option".

paragraph 3, line I: "The usual statistical information the number...."

paragraph 3, line 4: Insert a dash $(-)$ between "weight" and "is".

page 2,

panagraph 2, line 3: replace "Rather" with "For such information".

paragraph 2, line 4: "and the references therein".

paragraph 3, line 3: remove the hyphen.

page 3,

insert subheading: "Iteratively Reweighted Least Squares"

line 2: "...years. It is..."

page 4,

line 5 from top: equation shoud read

$=\left(\left(W^{(k+1)}\right)^{I / 2} A\right)^{+}\left(W^{(k+1)}\right)^{I / 2} b$.

line 4 from bottom: add a subscript 2 , to $\|\cdot\|$.

Should read: $\|\cdot\|_{2}$.

Insert after last line: "The function that is being minimized determines the formula for the weight function used. In general, we minimize $\sum_{i=1}^{m} \rho\left(r_{i}(x) / s\right)$ so that the weight function is given by a $W(u)=\rho^{\prime}(u)$.

page 5, line 2: Tuning Constant* (should have elavated asterisk). line 6 from top: function is $I /(I+|u / F|)$. 
page 5,

page 6 ,

page 8 ,

page 28, insert as footnote:

*

These are default values for the tuning constants which are designed to have $95 \%$ asymptotic efficiency with respect to ordinary least squares when the distrubances from the normal or Gaussian distribution and a scaling function converge to the standand deviation of that disturbance distribution.

insert subheading: "Selecting the Rark of the Data Matrix" paragraph 2, line 6: insert a comme after "occur". insert subheading: "Sore Numerical Results"

reference 4: replace "private cormunication, June 1976" with "Non-Iinear Least Squares and Equations," The State of the Art in Nurnerical Analysis, Accidemic Press, 1977. 